\title{
Regulation of the Contractile Activity of the Small Intestine Isolated Segment in Vitro
}

\author{
Ruslan Valerievich Savchuk ${ }^{1}$, Dmitriy Aleksandrovich Zhukovsky ${ }^{1}$, \\ Natalya Vladimirovna Shmatkova ${ }^{2}$ \\ ${ }^{1}$ Department of Urology and Nephrology, Odessa National Medical University, Odessa, Ukraine \\ ${ }^{2}$ Department of General Chemistry and Polymers, Odessa I. I. Mechnikov National University, Odessa, Ukraine \\ Email addres \\ savrus7@rambler.ru (R. V. Savchuk), dazhukovskij@gmail.com (D. A. Zhukovsky), nshmatkova@ukr.net (N. V. Shmatkova)
}

\section{To cite this article:}

Ruslan Valerievich Savchuk, Dmitriy Aleksandrovich Zhukovsky, Natalya Vladimirovna Shmatkova. Regulation of the Contractile Activity of the Small Intestine Isolated Segment in Vitro. International Journal of Pharmacy and Chemistry. Vol. 3, No. 5, 2017, pp. 62-66.

doi: $10.11648 /$ j.jppc.20170305.11

Received: May 29, 2017; Accepted: September 5, 2017; Published: October 17, 2017

\begin{abstract}
Radical cystectomy with urine derivation in the segment of the small intestine is the "gold standard" for the treatment of muscle-invasive bladder cancer. The main method of urine derivation, providing the best quality of life for the patient, is an orthotopic bladder formed from the ileal segment of the intestine. The aim of study was to examine the effect of new chemical compounds on the contractile activity of the ileal wall of mini-pigs in vitro. Pyrrole-2-carbaldehyde 3(dimethylamino) benzoylhydrazone and 1-methylisatin N, N-dimethylcarbamylhydrazone are two compounds with the greatest constrictor activity. Such compounds in concentration of $1 \times 10^{-3} \mathrm{~mol} / 1$, increase the contractile activity on $54.3 \pm 9.2 \%$ and $51.2 \pm 3.1 \%$, the amplitude of contractions on $59.01 \pm 12 \%$, and $49.18 \pm 14 \%$, the frequency of reductions on $47.9 \pm 6.4 \%$ and $31.3 \pm 5.3 \%$. Pyrrole-2-carbaldehyde 2-hydroxybenzoylhydrazone and isatin benzoylhydrazone reduce hypertonicity on $49.6 \pm$ $3.8 \%$ and respectively on $39.6 \% \pm 3.1 \%$. Pyrrole-2-carbaldehyde 2 -hydroxybenzoylhydrazone and isatin benzoylhydrazone are the two compounds that reduce hypertonicity on $49.6 \pm 3.8 \%$ and $39.6 \% \pm 3.1 \%$, making antispasmodic effect with tropicity to ileum.
\end{abstract}

Keywords: Radical Cystectomy, Neobladder, New Chemical Compounds, Proserin, Gynipral

\section{Introduction}

Radical cystectomy with urine derivation in the small intestine segment is the "gold standard" for the treatment of muscle-invasive and other forms of bladder cancer $[1,2]$. The main method of urine derivation, based on the analysis of scientific literature, providing the best quality of life for a patient and stable indicators of water-electrolyte metabolism, is an orthotopic urinary bladder, formed from the ileal segment [3]. The percentage of orthotopic ileocystoplasty reaches $66 \%$ in some specialized centers in Europe [4]. The segment of the ileum is resected to form a reservoir from the gastrointestinal tract $[5,6,7]$.

The segment is detubulated and then stitched together in $\mathrm{S}$, $\mathrm{W}, \mathrm{U}, \mathrm{M}, \mathrm{N}$ - anastomosis, turning into bowl-shaped reservoir, which increases the capacitance and decreases the intracavitary pressure $[8,9]$.

An artificial intestinal reservoir, previously detubulated and covered with intestinal epithelium, created to perform unnatural urodynamic properties, is not always able to provide satisfactory quality of urination.

The neobladder hyperactivity is observed in the early postoperative period due to connection with the reaction of the intestinal wall to a new aggressive environment (urine). The adaptation processes are stabilized in the further postoperative period and manifested with the hypoactivity of the neobladder, due to denervation of the nerve plexuses. Peristaltic rhythmic contractions of the smooth muscle wall of the gastrointestinal tract organs in mammals are provided by specific pacemakers -interstitial cells of Cajal (ICC). There are four types of ICC characteristic for this smooth muscle that differ among themselves in specific location, amplitude frequency and temporal characteristics of activity. Two types of pacemaker cells prevail in the intestine: ICC-SM located in the submucosal part, in the Maisner plexus and ICC-MY cells located in Auerbach plexus. These pacemakers generate an 
independent pattern of rhythm activity independently of each other. ICC-MY causes low-amplitude contractions with high frequency (10-15 contractions per minute), and ICC-SM causes high-amplitude with low frequency (0.5-1.5 contractions per minute). Most of the experimental invetigations have been carried out on mammals (rats, pigs), given that the gastrointestinal tract under investigation resembles the human intestine in its properties. It can be used for interpretation the results of the contractile regulation of the smooth muscles of the human intestine [10].

There are no recommendations and theoretical developments, based on the full information in the available literature, about the pharmacological correction of the neobladder contractile function at different stages of the postoperative period.

The aim of study was to examine the effect of new chemical compounds on the contractile activity of the ileal wall of mini-pigs in vitro.

\section{Materials and Methods}

\subsection{In Vitro Studies}

The experiments were performed on isolated strips of the small intestine of mini-pigs, which were fixed in the working chamber from one side to the stationary hook, and on the other to the load cell stem. The strips were isolated from the terminal section of the ileum $2 \mathrm{~cm}$ up to ileocecal angle with 10 to 15 $\mathrm{mm}$. in length and $4-5 \mathrm{~mm}$ in width. All preparations fixed in the experimental chamber were perfused for 30 minutes at $37^{\circ} \mathrm{C}$ with Krebs-Henseleit salt solution of the following composition (mol / 1): sodium chloride (118.4), potassium chloride (4.7), calcium chloride (2.5), magnesium sulfate (1.2), potassium dihydrogen phosphate $(1,2)$, sodium bicarbonate (24.9), glucose (11.1), followed by another 90 minutes with a saline solution with a potassium chloride concentration of 60 mmol / 1. The working solutions were saturated with a gas mixture of $95 \%$ oxygen and $5 \%$ carbon dioxide.

The chemically pure reagents of the company "Sinbias" (Ukraine), which were dissolved in distilled water, used to prepare all Krebs buffer solutions. The automatic thermostat made it possible to maintain the temperature of the solutions in the chamber at $37^{\circ} \mathrm{C} \pm 0.5^{\circ} \mathrm{C}$. The contractile activity was investigated in isometric mode using a force sensor. Signals were recorded using an analog-to-digital converter. The investigation consists of two series of experimental studies and analysis of the data. A screening of 22 compounds was performed, with the potential to stimulate and relax the smooth muscles. The choice of compounds was carried out according to the SAR (Structure Activity Relationship) method, using the principle of the greatest structural similarity with the known blockers of anticholinesterase and spasmolytic compounds, among the basic structural classes of hydrozone derivatives. Proserine and ginipral were used to control the effect of new chemical compounds.

The constrictive properties of the new hydrozone derivatives were studied in the first series of experiments, in comparison with proserin during reaction on the isolated ileal intestinal segment in experimental animals in vitro.

\subsection{Experimental Research}

The Second Series of Experiments Consisted in Studying the Spasmolytic properties of the test compounds on smooth intestinal musculature in vitro in comparison with ginipral, against the background of stimulation of $\mathrm{KCl}$. The administered volume of the $\mathrm{KCl}$ solution was $0.1-1.15 \mathrm{ml}$, with a concentration of $60 \mathrm{mmol} / \mathrm{l}$ until a rhythmic, high amplitude contraction was obtained.

The test compounds were used in the form of aqueous solutions, in the range of molar concentrations $1 \times 10^{-1}-1 \times 10^{-7}$ mol/l. They were prepared by dilution with $1 \times 10^{-1} \mathrm{M}$ solutions of the corresponding compounds. The volume of them was $209 \mathrm{ml}$.

The compounds were synthesized at the Department of General Chemistry and Polymers in Odessa I. I. Mechnikov National University. Their composition and structure is determined by a combination of physical and chemical methods of investigation.

\section{Results of the Research}

Screening of the constrictive properties of new chemical compounds has demonstrated the prospectivity of the study and the greatest effectiveness of the action of three chemical compounds: pyrrole-2-carbaldehyde 3- (dimethylamino) benzoylhydrazone (P2C3DBh, compound I ); 1-methylisatin $\mathrm{N}, \mathrm{N}$-dimethylcarbamylhydrazone (1MDCh, compound II) and 4- (dimethylamino) benzaldehyde N, Ndimethylcarbamylhydrazone (4DCh, compound III). The spontaneous contractions of the ileal intestinal segment were recorded in the control group, the avarage frequency of which was $0.48 \pm 0.02 \mathrm{~Hz}$. and the amplitude $0.61 \pm 0.7 \mathrm{~g}(\mathrm{n}=16)$.

The best studied properties, according to the in vitro experiment, were exhibited by compounds I and II. An increase in contractile activity on $12.5 \pm 2.1 \%$ was observed in concentration of $1 \times 10^{-5} \mathrm{~mol} / 1$ when compound I was injected into the working chamber. A concentration of $1 \times 10^{-4} \mathrm{~mol} / 1$ led to an increase in the contractions on $35,1 \pm 4.5 \%$. The contraction was increased on $54.3 \pm 9.2 \%$, with the concentration of $1 \times 10^{-3}$ $\mathrm{mol} / \mathrm{l}$ and the further increase in concentration did not change the strength of ileum strips in vitro.

Compound II exhibited similar concentration-dependent activity by enhancing the contractile force on $51.2 \pm 3.1 \%$, in concentration of $1 \times 10^{-3} \mathrm{~mol} / 1$. The further increase in concentration did not lead to a change in contractile activity.

Proserin, as an effective inhibitor of cholinesterase, stimulated a $100 \%$ reduction in a concentration of $1 \times 10^{-3} \mathrm{~mol} / 1$.

Serum concentrations are not toxic, as the tissue is quickly restored to its original values after washing and after repeated applications of even higher concentrations.

The results of the effective minimum dosage of I and II compounds, allowed to study their effect on the amplitude, frequency of spontaneous contractions, and tonic tension in the ileum of the mini-pigs. 


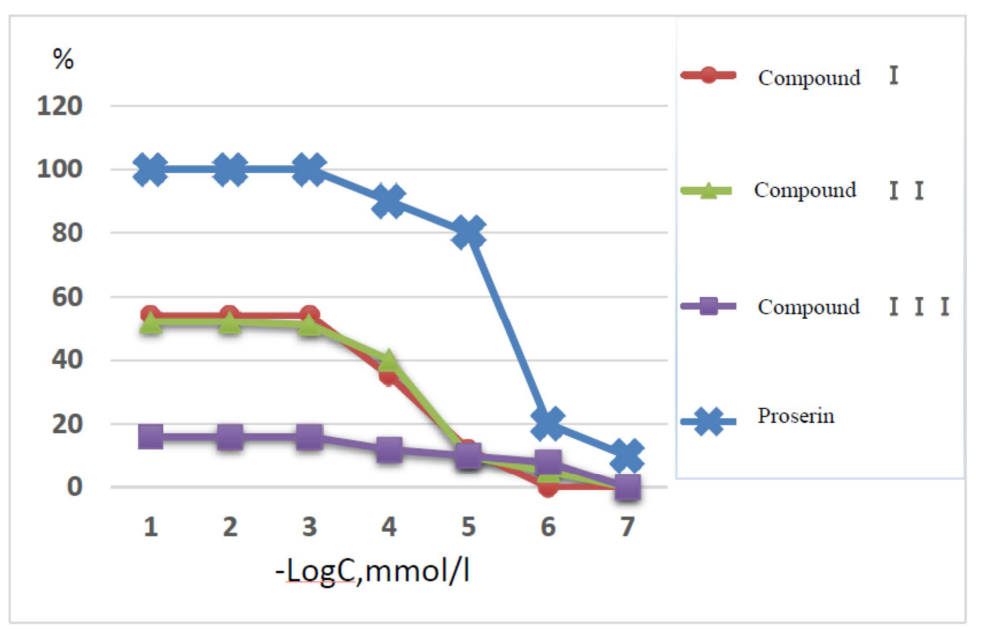

Figure 1. The constrictive effect of the test substances on ileum strips in vitro.

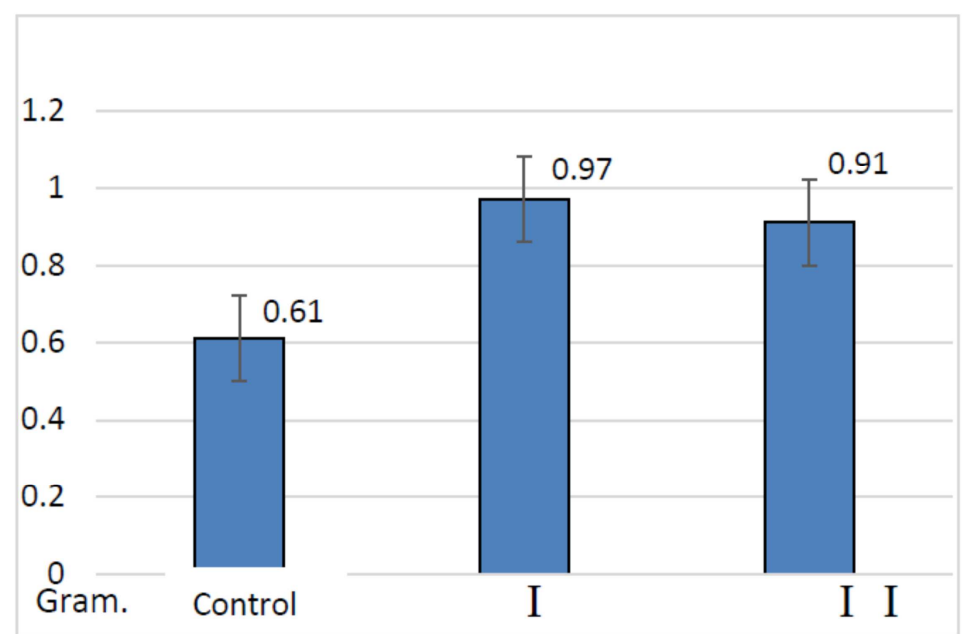

Figure 2. Dynamics of amplitude variation (g) of contractions under the influence of compounds I and II, in a concentration of $1 \times 10^{-3}$ mol $/ \mathrm{l}$. (N $=14$, $p \leq 0.05)$.

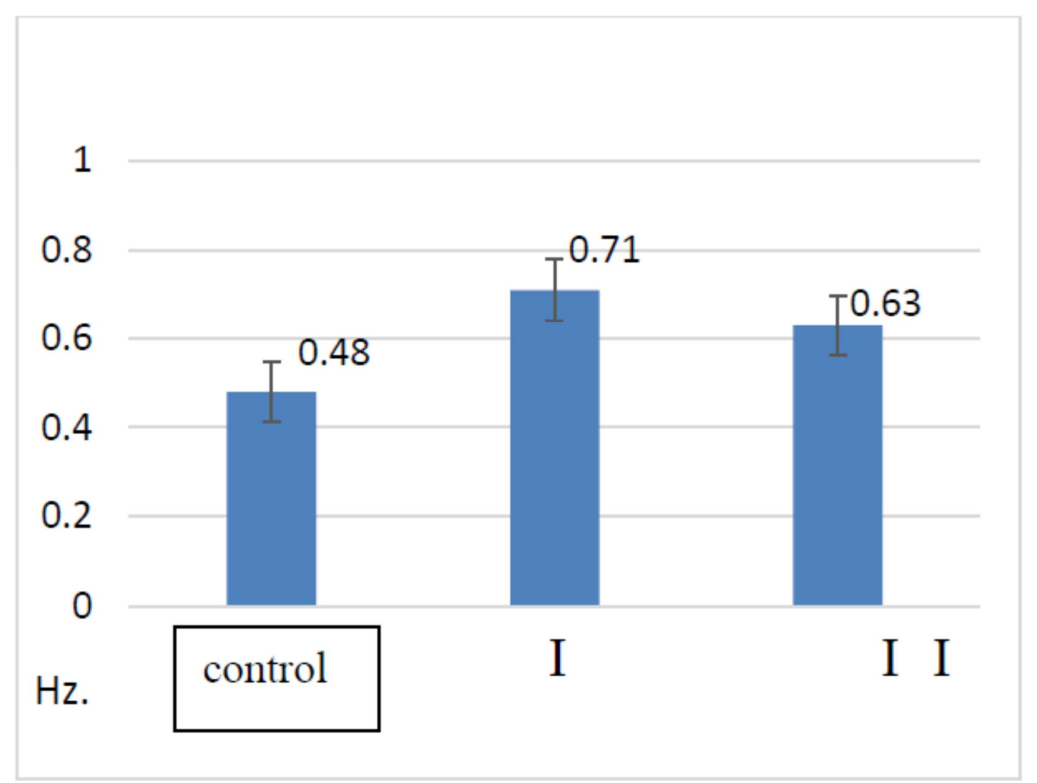

Figure 3. Dynamics of changes in the frequency of contractions $(\mathrm{Hz})$ under the influence of compounds I and II, in a concentration of $1 \times 10^{-3}$ mol/l. $(\mathrm{N}=14$, $p \leq 0.05)$. 
Compound I increases the amplitude of the contractions on $59.01 \pm 12 \%$ and compound II raises the amplitude on 49.18 $\pm 14 \%$, in comparison with the control.

The frequency of contractions also increased under the influence of the test compounds, as compound I increased the frequency on $47.9 \pm 6.4 \%$, and compound II on $31.3 \pm 5.3 \%$.

The tonic stress under the action of compound I increased on $68 \pm 13.2 \%$, and $53 \pm 5.7 \%$ with using of compound II.

These properties of the test compounds may indicate a constrictive ability and the prospect of their use in correcting the hypoactivity of the neobladder. Screening of the spasmolytic properties of the test compounds showed pronounced desired properties in two compounds: pyrrole-2carbaldehyde 2-hydroxybenzoylhydrazone (PChBh) and isatin benzoylhydrazone (IBh). The mini-pigs ileal intestinal strips were perfused with a hypercalic solution with a $\mathrm{KCl}$ concentration of $60 \mathrm{mmol} / \mathrm{l}$, with a steady increase in the basal tone of the detrusor strips. The spasmolytic effects of the test substances were studied with the addition of a hypercalic solution. The results of the effective action of substances $\mathrm{PChBh}$ and IBh in comparison with ginipral, exhibiting spasmolytic activity, are presented in the concentration-effect graph Figure 4.

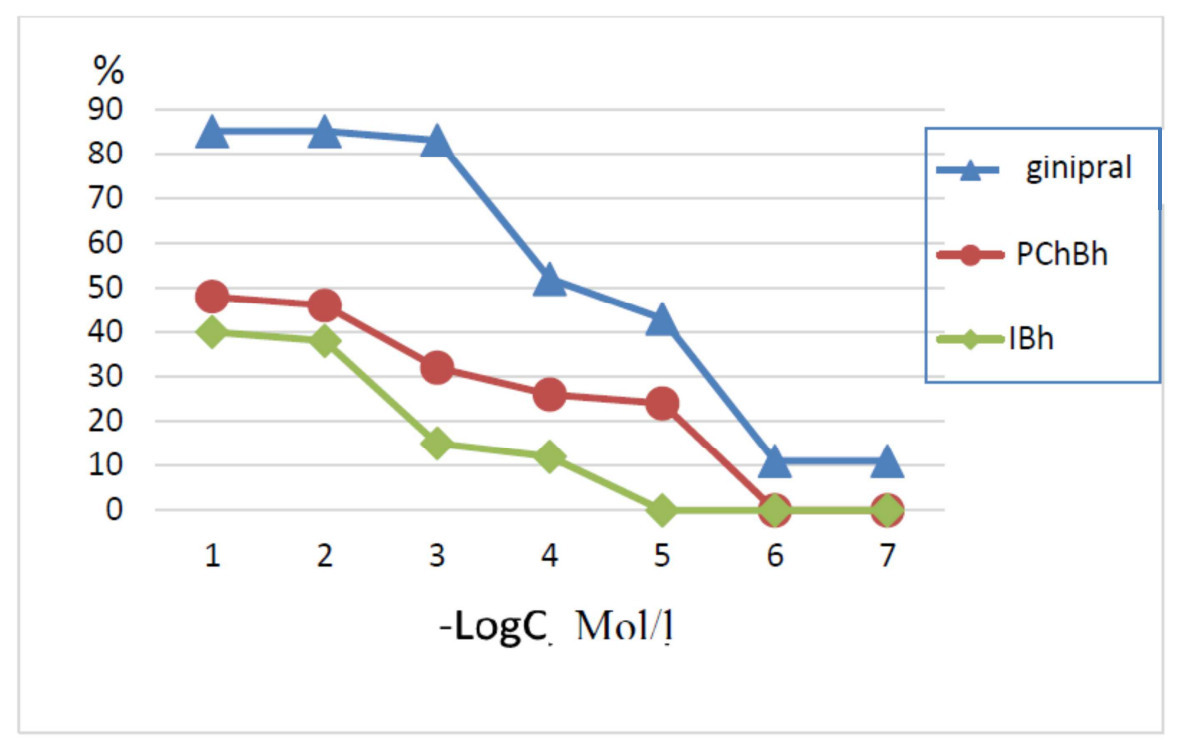

Figure 4. Spasmolytic properties of test substances on ileum strips in vitro $(n=16, p \leq 0.05)$.

As a result of the in vitro experiment, it was established that the $\mathrm{PChBh}$ compound exerts a spasmolytic effect on the detrusor strips against the background of $\mathrm{KCl}$ stimulation of $60 \mathrm{mmol} / \mathrm{l}$, decreasing the tone in a concentration of $1 \times 10^{-5}$ $\mathrm{mol} / 1$ on $23.4 \% \pm 4.2 \%$ and exhibits a persistent effect in concentration of $1 \times 10^{-2} \mathrm{~mol} / 1$, reducing the tone on $49.6 \pm$ $3.8 \%$. IBh compound depresses hypertonus ileum in concentration of $1 \times 10^{-4} \mathrm{~mol} / \mathrm{l}$, decreasing the tone of the ileum band on $11.3 \% \pm 0.9 \%$. The stable effect was manifested in a concentration of $1 \times 10^{-2} \mathrm{~mol} / 1$, reducing the hypertonicity on $39.6 \% \pm 3.1 \%$.

Ginipral began to show spasmolytic abilities in concentration of $1 \times 10^{-7} \mathrm{~mol} / 1$, reducing the contractile activity on $10.5 \% \pm 0.6 \%$, and in concentration of the solution $1 \times 10^{-2} \mathrm{~mol} / 1$ reduced it on $84.1 \% \pm 5.1 \%$. A further increase in its concentration did not lead to a decrease in the contractile activity of isolated samples of the bladder.

\section{Conclusions}

As a result of experimental research, new chemical compounds have been shown to affect the contractile activity of the ileum segment in vitro. The two compounds among them, show the greatest constrictor activity: pyrrole-2- carbaldehyde 3- (dimethylamino) benzoylhydrazone (P2C3DBh, compound I) and 1-methylisatin N, Ndimethylcarbamylhydrazone (1MDCh, compound II) in concentration of $1 \times 10^{-3} \mathrm{~mol} / 1$.

Two compounds with properties of tendency to ileum which reduce hypertonicity on $49.6 \pm 3.8 \%$ and $39.6 \% \pm$ $3.1 \%$ can be distinguished on: pyrrole-2-carbaldehyde 2hydroxybenzoylhydrazone (PChBh) and isatin benzoylhydrazone (IBh). The new chemical compounds with antispasmodic and constrictive properties identified and require further investigation of the mechanism of action and effect on various links of the pathogenesis of ileum reduction and study with in vivo experimental conditions.

\section{References}

[1] Park J. 1. Radical cystectomy and orthotopic bladder substitution using ileum. Ahn H. Korean J Urol. 2011 Apr; 52(4):233-40. Epub 2011 Apr 22.

[2] Stein J. P. Radical cystectomy in the treatment of invasive bladder cancer: long-term results in 1054 patients /G. Lieskovsky, R. Cote [et al.] // J. Clin. Oncol. - 2001. - Vol. 19. - P. 666-775; 486-492; discussion 491-492. 
[3] Hautmann R. E. 25 years of experience with 1,000 neobladders: long-term complications / R. E. Hautmann, R. C. de Petriconi, B. G. Volkmer // J. Urol. - 2011. - Vol. 185. - P. 2207-2212.

[4] Stein J. P., Skinner D. G. Application of the T-mechanism to an orthotopic (T-pouch) neobladder: a new era of urinary diversion. World J Urol. 2000;18:315-323.

[5] Campbell's Urology / Edited by P. C. Walsh [et al.]. - 8th ed. - Philadelphia : W. B. Saunders Co., 2002. - Vol. 3. - 3420 p.

[6] Cystectomy for transitional cell carcinoma of the bladder: results of a surgery only series in the neobladder era / R. E. Hautmann, J. E. Gschwend, de Petriconi R. C. [et al. // J. Urol. - 2006. - Vol. 176. - P.486-492.

[7] Hollenbeck BK, Miller DC, et al. Aggressive treatment for bladder cancer is associated with improved overall survival among patients 80 years old or older. Urology. 2004;64:292-7.

[8] Singh V. 1. Urodynamic and continence assessment of orthotropic neobladder reconstruction following radical cystectomy in bladder cancer; a prospective, blinded North Indian tertiary care experience. /Mandal S.1., Patil S.1., Sinha R. J. et all. / South Asian J. Cancer. 2014 Oct;3(4):223-6.

[9] Hautmann R. E. ICUD-EAU International Consultation on Bladder Cancer 2012: Urinary diversion Abol-Enein H, Davidsson T, Gudjonsson S, Hautmann SH, Eur Urol. 2013 Jan; 63(1):67-80. Epub 2012 Aug 31.

[10] Blair P. J., Rhee P.-L., Sanders K. M., Ward S. M. The Significance of Interstitial Cells in Neurogastroenterology. J. Neurogastroenterol. Motil, 2014; 20, 3: 294-317. 